\title{
In Situ Biomimetic Synthesis of Gelatin-Carbon Nanotube-Hydroxyapatite Biocomposites as Bone Filler
}

\author{
MAHSHID ASLANI ${ }^{1}$, MASOUMEH MESKINFAM ${ }^{2 *}$ and HAMID REZA AGHABOZORG ${ }^{3}$ \\ ${ }^{1}$ Chemistry Department, North Tehran Branch, Islamic Azad University, Tehran, Iran. \\ ${ }^{2}$ Chemistry Department, Lahijan Branch, Islamic Azad University, Lahijan, Iran. \\ ${ }^{3}$ Catalyst and Nanotechnology Division, Research Institute of Petroleum Industry, Tehran, Iran. \\ ${ }^{*}$ Corresponding author E-mail: meskinfam @ gmail.com
}

http://dx.doi.org/10.13005/ojc/330127

(Received: December 26, 2016; Accepted: February 07, 2017)

\begin{abstract}
Inorganic-polymeric composites are promising materials for bone repair; the so called biomimetic approach leverages the interaction between polymer and inorganic mineral and generates the appropriate material features. In this work, gelatin multiwalled carbon nanotube (MWCNT) hydroxyapatite $(\mathrm{HA})$ biocomposites containing different concentration of gelatin were prepared by in situ biomimetic approach. The synthesized composites were characterized using X-ray diffraction (XRD), Fourier transform infrared spectroscopy (FT-IR) and scanning electron microscopy (SEM). The results showed that, gelatin is a suitable matrix for nucleation and growth of hydroxyapatite nanocrystals and homogenous dispersion of nanotubes. Mesenchymal Stem cells (MSCs) culturing on composites and MTT assays were used to determine the in-vitro biocompatibility of the synthetized biocomposides, which showed no negative effect on the cell viability and proliferation. The experimental work has demonstrated that increasing gelatin concentration can lead to the formation of more uniform HA spherical particles and enhance the biocompatibility of the composites.
\end{abstract}

Keywords: Gelatin, Biocompatibility, Hydroxyapatite, bone filler.

\section{INTRODUCTION}

Bone is a natural composite material consisting of organic phase containing- type-I collagen and inorganic phase named hydroxyapatite $(\mathrm{HA})$ with a chemical formula of $\mathrm{Ca}_{10}\left(\mathrm{PO}_{4}\right)_{6}(\mathrm{OH})_{2}$ that can enhance osteoconductivity.

In presence of a defect in the bone structure, the need for synthetic bone grafts may arise, depending on the complication of the bone defect. If the damage is minor, the bone tissue has its own capability to self-regenerate within a few weeks. As the severity of the defect increase, using composites as bone filler can be helpful, and in the case of severe injuries with significant loss of volume, the bone would not heal by itself and scaffold grafting is required to restore the bone function without damaging living tissues. 
Bone tissue engineering benefits of the use of tailored biomaterials to improve bone regeneration without producing side effects in the human body ${ }^{1-2}$. An accurate selection of the most appropriate biomaterials for bone regeneration is very important: biocompatibility, biodegradability and the ability of mimicking the structure and chemical properties of extracellular matrix (ECM) are some of the desired characteristics $^{3-5}$.

In recent years, many attempts have been focused on the development of materials and techniques for imparting the suitable biological properties to the synthetic biocomposites, to be used in the replacement of the natural bone ${ }^{2,6}$. One of the main methodologies developed in the bone regeneration field is the biomimetic approach using natural or synthetic polymeric templates via an in situ mineralization method ${ }^{5,-10}$. So, in recent years, the use of embedded inorganic nanoparticles in polymeric matrixes has attracted the interest of researchers, because of the similar composition of such particles to natural bone as well as their good biocompatibility ${ }^{5,11,12}$. Different natural and synthetic polymers $^{2,9,13-15}$ have been applied as templates for the synthesis of composites via biomimetic method. Gelatin as a natural water-soluble biopolymer, is a hydrolysis product of collagen and is expected to be suitable for hard tissue applications due to its biological functional groups ${ }^{16}$. Apart from the brittleness of nanostructured $\mathrm{HA}(\mathrm{nHA})$ that limits its application, it has a higher surface area compare to $\mathrm{HA}$, and consequently higher reactivity, and can enhance Mesenchymal Stem Cells (MSCs) adhesion and proliferation. Thanks to the different properties of such materials, the combination of $\mathrm{nHA}$ with a polymer such as gelatin can lead to the formation of a suitable composite for hard tissues regeneration ${ }^{2,17-19}$. The properties of gelatin HA composites in terms of biocompatibility, degradation and osteoconductivity making them suitable candidate for applications in hard tissue reconstruction that have been reported in several works ${ }^{2,14,20}$. Multiwalled carbon nanotubes (MWCNTs) due to their small dimensions, high aspect ratio (length to diameter), high strength and stiffness can be an ideal reinforcement material that would improve the composite characteristics, without diminishing its bioactivity ${ }^{16,21-23}$.
The aim of this work is the preparation of gelatin- multiwalled carbon nanotube- hydroxyapatite biocomposites as a bone filler via in situ biomimetic method and the evaluation of gelatin concentration alteration effect on biocompatibility behavior of the prepared nanocomposites.

\section{MATERIALS AND METHODS}

\section{Materials}

Gelatin (Type B, powder, bovine skin), calcium nitrate tetrahydrate $\mathrm{Ca}\left(\mathrm{NO}_{3}\right)^{2}, 4 \mathrm{H}_{2} \mathrm{O}$ and diammonium hydrogen phosphate $\left(\mathrm{NH}_{4}\right) 2 \mathrm{HPO}_{4}$ were purchased from Sigma - Aldrich and Carboxyliated multiwalled carbon nanotube (outer diameter 8-10 $\mathrm{nm}$, length 10-30 $\mu \mathrm{m}$ ) was provided from Neotec Co. (Iran). All chemicals were used without any further purification.

\section{Experimental procedures \\ Preparation of GEL-MWNTs-HA composites by in situ biomimetic method}

For studying the effects of biopolymer concentration on the final structure and biocompatibility of composites, different weight of gelatin, 0.24, 0.36 and $0.48 \mathrm{~g}$, were dissolved in $50 \mathrm{ml}$ of deionized water and stirred at $45^{\circ} \mathrm{C}$, until solutions became apparently transparent. Then the desired amount of MWCNT (4 wt. \%) was sonicated in ethanol for 10 min. Subsequently, gelatin solution was added into the MWCNT dispersion and the obtained mixture was sonicated for 1.5 hours. Prepared composites containing $0.24,0.36$ and $0.48 \mathrm{~g}$ of gelatin, named by codes $\mathrm{S}-1, \mathrm{~S}-2$ and $\mathrm{S}-3$, respectively. Then $\mathrm{Ca}$ $\left(\mathrm{NO}_{3}\right)_{2} \cdot 4 \mathrm{H}_{2} \mathrm{O}$ and $\left(\mathrm{NH}_{4}\right)_{2} \mathrm{HPO}_{4}$ were added to the gelatin-MWCNT mixtures, so that $\mathrm{Ca} / \mathrm{P}$ molar ratio 1.67 was obtained. After complete dissolving the salts under agitation, ammonia solution was added to the mixture for adjusting the $\mathrm{pH}$ at 10.2 and promoting the HA nucleation. The obtained samples were kept overnight, filtered, and washed with deionized water until the filtrate neutralized $(\mathrm{pH}=7)$. Pure HA was prepared as mentioned above by omission of gelatin and MWCNT steps.

The prepared products were characterized using Fourier Transform Infrared Spectroscopy (FTIR, PerkinEImer 400, USA), X-ray Diffraction (XRD, PW 1800 Philips, China) and Scanning Electron Microscopy (SEM, EM 3200, KY, China). 


\section{In vitro biocompatibility test}

Biocompatibility is the compatibility of a product with a biological system. The purpose of biocompatibility tests is the determination of the product suitability for human use and the estimation of its potential for harmful physiological effects. For this purpose, Mesenchymal Stem Cells (MSCs) maintained from the ophthalmic research center of Shahid Beheshti University (Iran) and were used. In MTT assay, MWCNTs, gelatin, HA, S-1, S-2 and S-3 were cultured with MSCs for 1, 3 and 7 days, and a culture without composite was used as a blank control group. Defreeze MSCs were transferred into culture flasks containing Dulbecco's Modified Eagles Medium (DMEM), 20\% fetal bovine serum and $1 \%$ antibiotics (100 ig/ml penicillin and $100 \mathrm{ig} /$ $\mathrm{ml}$ streptomycin). Changing the medium was taken place every 3 days. For cell culture experiments, sterilization of prepared samples were carried out via ethanol series and Phosphate Buffered Saline (PBS) solution, then they incubated in the culture media. The samples were seeded with MSCs $\left(10^{3}\right.$ cells $/ \mathrm{cm}^{2}$ ) by direct pipetting of the cell suspension onto the biocomposites and incubated in cell culture medium at $37^{\circ} \mathrm{C}$ and $5 \% \mathrm{CO}_{2}$. MSCs cultured without samples were used as a control group. For each composite type, three replicates were used. MTT assay (3- [4, 5-dimethylthiazol-2-yl]-2, 5-diphenylte-
$2 \mathrm{H}$-tetrazolium bromide) (Sigma-Aldrich) was applied for evaluation of MSCs proliferation on samples and control. After seeding at each time point, cells were incubated in $100 \mu \mathrm{l}$ MTT solution at $37{ }^{\circ} \mathrm{C}$ and $5 \% \mathrm{CO}_{2}$ for $3 \mathrm{~h}$. Then, $100 \mu \mathrm{l} /$ well of dimethyl sulfoxide (DMSO) was added and mixed after removing supernatants. When blue crystals of formazan dissolved completely, the absorbance of each well content was measured at $540 \mathrm{~nm}$ with Elisa reader (BioTek Co.).

\section{Statistical analysis}

Student's t-test was conducted to determine statistical significance among groups, and statistical significance was considered at $p<0.05$.

\section{RESULTS AND DISCUSSIONS}

\section{Chemical and morphological characterizations}

A wide research efforts have been carried out to prepare suitable organic-inorganic composites by mimicking the natural process of bone repair. Inorganic mineral formation, size, growth, morphology and uniform distribution into organic template are the critical parts of biomimetic synthesis approach ${ }^{15}$. In the last 8 years, we have prepared different composites via in situ biomimetic methods using biopolymers such as

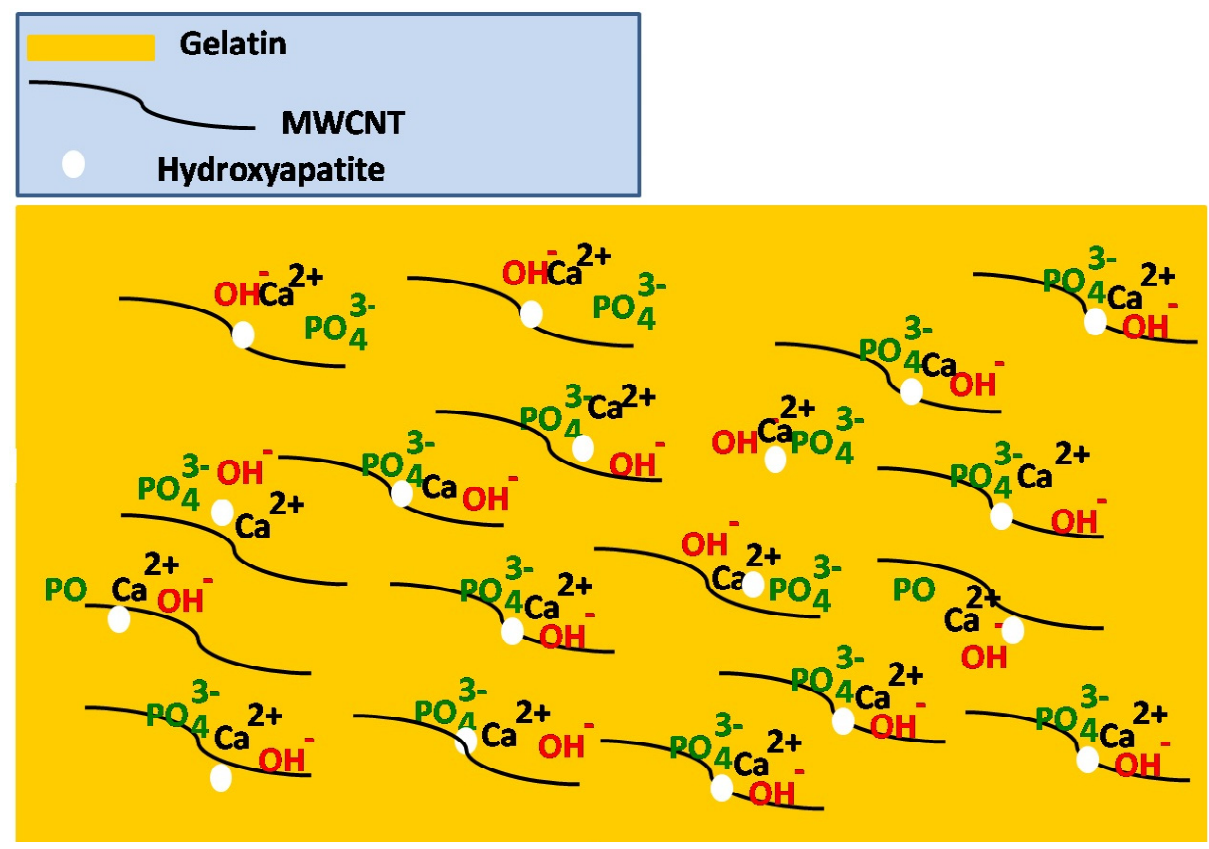

Fig.1: Schematic of gelatin- MWCNTs-HA composite preparation 


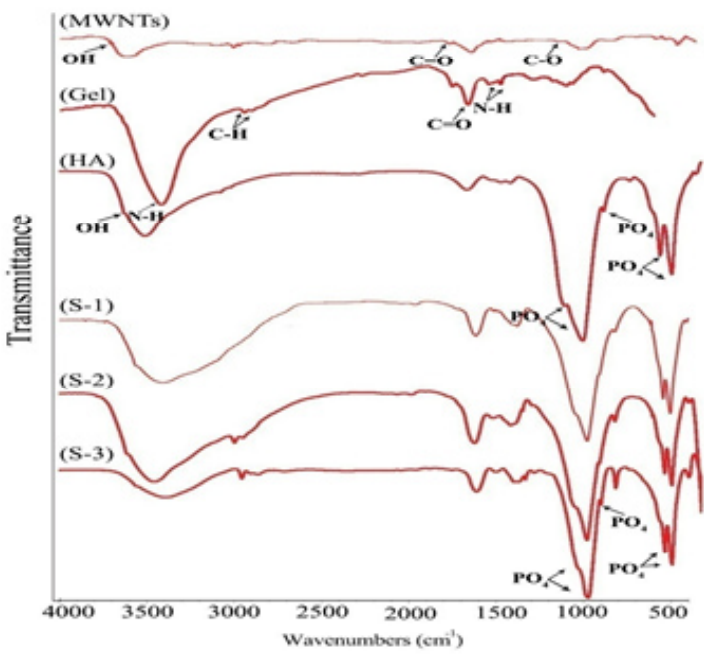

Fig. 2: FTIR spectra of MWCNTs, gelatin, HA, S-1, S-2 and S-3

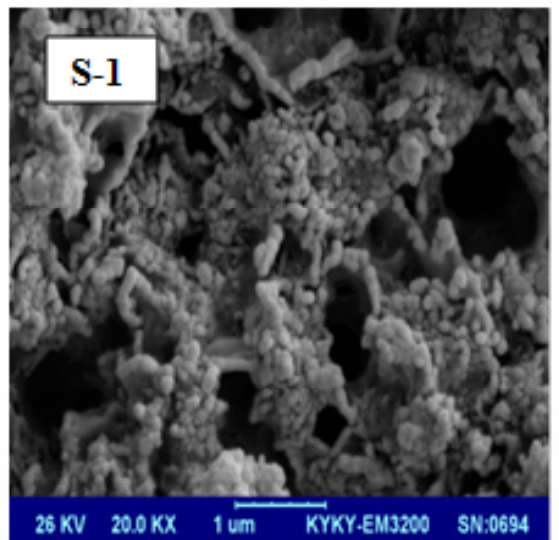

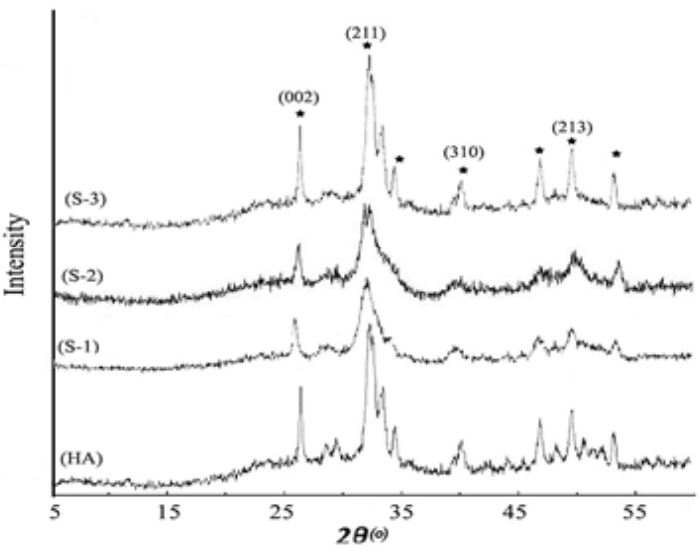

Fig. 3: XRD patterns of (a) HA, S-1, S-2 and S-3
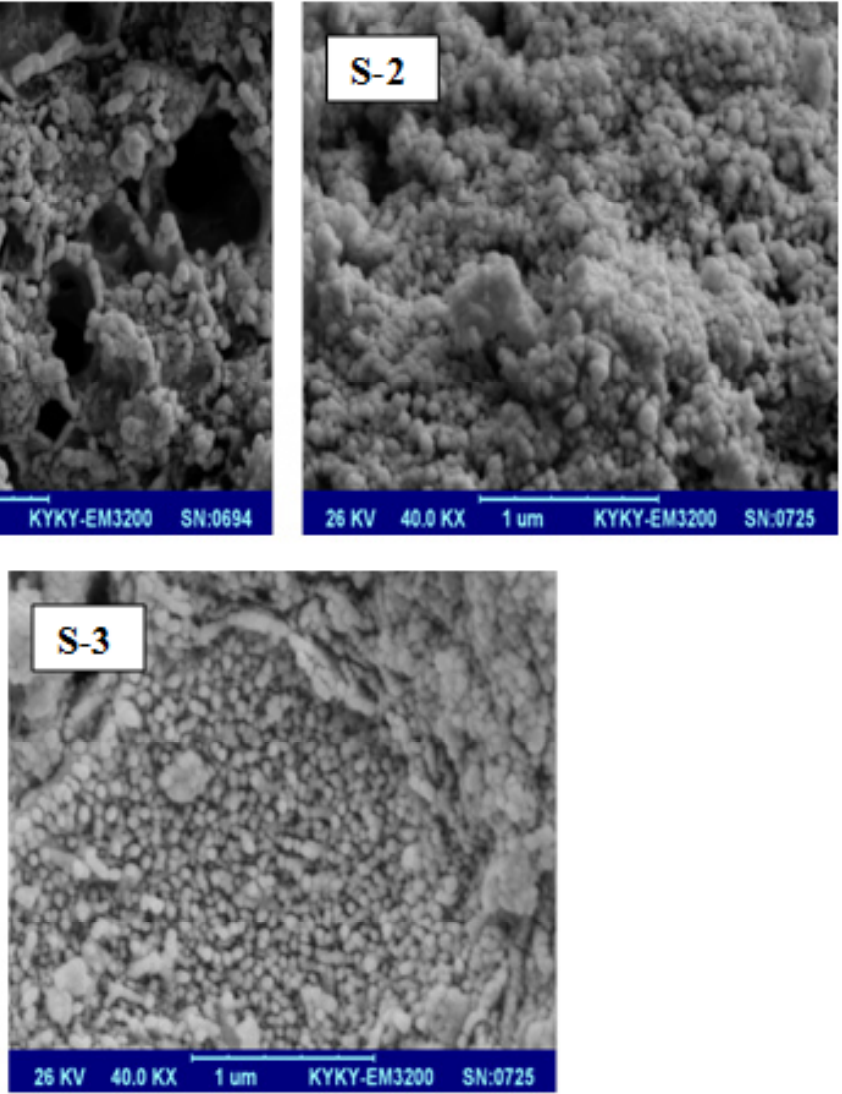

Fig. 4: SEM images of S-1, S-2, S-3 
pectin, starch, alginate and gelatin as a bone filler ${ }^{2}$, $5,9,11$. In this work, we designed new composites of gelatin-MWCNTs-HA by leveraging the suitable bioactivity and biocompatibility properties of gelatinHA nanocomposites ${ }^{2,5}$, the excellent mechanical properties of $\mathrm{CNTs}^{25}$ and the positive effects of functionalized CNTs on cell growth, biocompatibility, bio mineralization and bone formation ${ }^{13,25}$. The aim was using negative functional groups of MWCNTs and gelatin as active binding sites for in situ nucleation of $\mathrm{HA}$ in polymeric matrix template that leads to biocomposites formation. Ultrasonic method has been exploited the dispersion of MWCNTs in the gelatin matrix to conquer van der Waals interactions between CNTs and create positive charges on the carboxyl groups of MWCNTs that can act as electrostatic attraction sites for negative functional groups of gelatin ${ }^{13}$. As it is shown schematically in Fig. 1, in situ precipitation of HA was induced in this step due to bonding of the calcium ions with negative functional groups of MWCNTs and gelatin that promote HA nucleation in basic media.

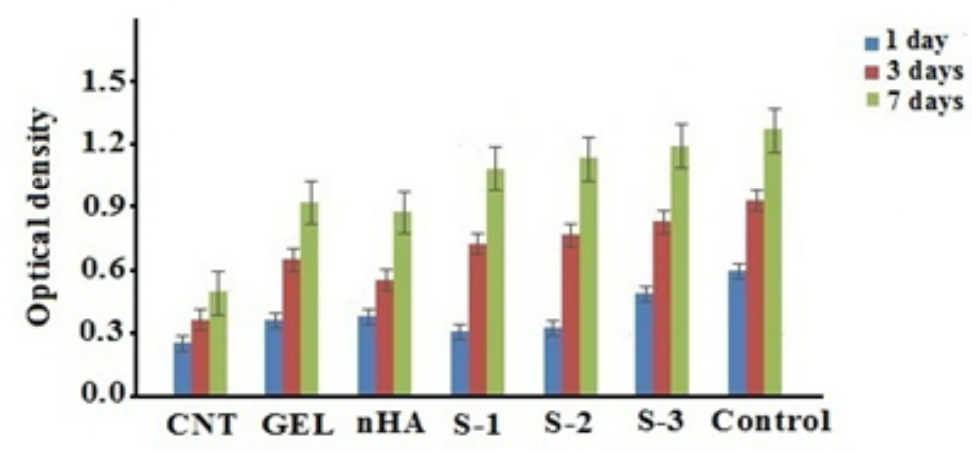

(b)

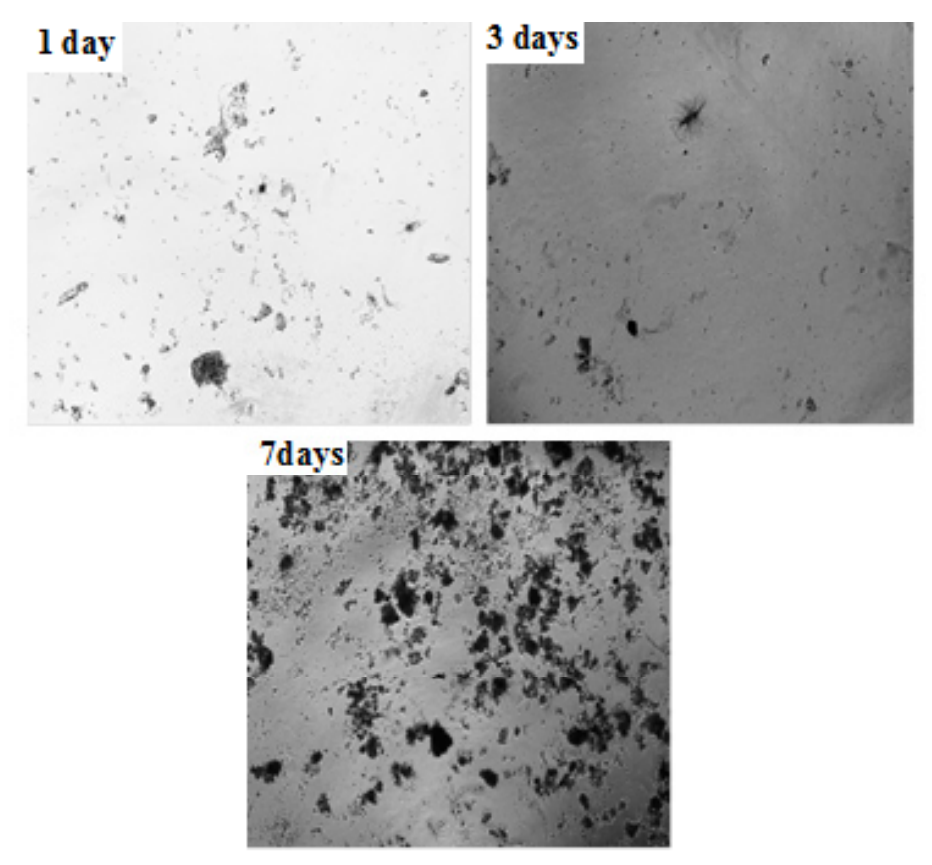

Fig. 5: (a) Cell proliferation of MSCs on MWNTs, gelatin, HA and nanocomposites by MTT assay and (b) optical microscopic image of S-3 after in vitro culture for 1, 3 and 7 days 
Fourier Transform Infrared Spectroscopy (FTIR)

Fig. 2 shows the FT-IR spectra of MWCNT, gelatin, HA and composite samples (S-1, S-2 and S-3). In MWCNT, bands at 1120, 1720, can be attributed to the $\mathrm{C}-\mathrm{O}, \mathrm{C}=\mathrm{O}$ stretching of carboxylic acids whereas bending and stretching vibration of hydroxyl group presented peaks at 672 and 3729 $\mathrm{cm}^{-1}$, respectively [13]. Gelatin spectra showed the bands at $3438 \mathrm{~cm}^{\prime 1}$ and $3423 \mathrm{~cm}^{\prime 1}$ assigned to the $\mathrm{OH}$ and $-\mathrm{NH}$ stretching of secondary amide while peaks at 2922 and $1640 \mathrm{~cm}^{-1}$ corresponded to the $\mathrm{C}-\mathrm{H}$ and the $\mathrm{C}=\mathrm{O}$ stretching. The observed bands between $1500 \mathrm{~cm}^{\prime 1}-1550 \mathrm{~cm}^{\prime 1}$ and the one at 670 $\mathrm{cm}^{\prime \prime}$ can be related to bending and out-of-plane wagging of secondary amide $-\mathrm{NH} 2^{2,24]}$. In the $\mathrm{HA}$ spectrum, absorbance bands at 472, 605, 961 and $1050 \mathrm{~cm}$ "1 corresponded to different vibration modes of phosphate group. The stretching and bending vibration modes of the $\mathrm{OH}$ presented the peaks at 3569 and $631 \mathrm{~cm}^{\prime 1}$, respectively 2, 5, 9, 11 .

FT-IR spectra of composites presented the same characteristics of the functional groups of gelatin, MWCNT and HA with a slightly shift to higher energy that can be the sign of bond formation between calcium ions and following phosphate ions that lead to HA nucleation along the MWCNTs length and in the gelatin matrix.

\section{X- ray Powder Diffraction (XRD)}

Fig. 3 shows the $X$-ray diffraction patterns of HA, S-1, S-2 and S-3. The peak positions for all patterns are matching closely to the diffraction peaks of mono phase HA (verified by JCPDS No. 9-432). Three main assignable peaks to (002), (211) and (310) plane of crystalline HA can be seen at 26.3, 32.2 and $39.9^{\circ}$ (2è), respectively. So, this method of composite preparation appears to be preferable to other in situ precipitation procedures, because there is no impurity of the different calcium phosphate phases and just pure single phase HA is produced in polymer matrix.

Increasing the gelatin amount in the composites lead to HA crystallinity increase so that characteristic peaks of HA can be completely distinguishable. This result confirms that the functional groups of gelatin in addition to MWCNTs have positive effect on HA nucleation. The presence of broad peaks in the XRD patterns of composites is related to the small particle size and low crystalline $\mathrm{HA}$ in the biopolymer matrix which is similar to natural bone ${ }^{9}$.

\section{Scanning Electron Microscopy (SEM)}

Fig. 4 shows the SEM images of S-1, S-2 and S-3. As it is observable, hydroxyapatite spherical nanoparticles are uniformly distributed on the gelatin and MWCNT matrix. In all images, no separation between organic and inorganic phase is observed. It appears that, as the gelatin concentration in the composites is increased, the homogeneity of HA spherical particles increases, while their size decreases.

This trend is due to the availability of more gelatin functional groups as a result of the increased polymer concentration that leads to HA nucleation in a more ordered pattern and causes the porous morphology turn to a more compact structure without any pores.

\section{Cell Culture Study}

For the evaluation of cytotoxicity effects, cell viability and proliferation on biocomposites, MTT assay was used. The results in Fig. 5a indicate that, at the first day, there is significant differences between absorbance values of composite S-3 compared to control group and their raw components $(P<0.05)$. The cells on composites, control group and raw components started to proliferate rapidly from the third day that is more remarkable for S-3 and control. At 7 days, the cell viability of S-3 had no significant differences $(P>0.05)$ whereas $S-1$ and $\mathrm{S}-2$ showed significant difference $(\mathrm{P}<0.05)$. From MTT results, it can be concluded that composites have no cytotoxic effects and that increasing the gelatin content (that lead to the formation of more homogenous nHA particles), has a positive effect on biocompatibility. Fig.5b presents the optical microscopic images of S-3 after cell culture. It shows the increasing of formazan crystals formation by passing time from one to seven days that can be the sign of alive cells presence and good biocompatibility of the composite. The cell culture results (Fig. 5a-b), compared to our pervious study ${ }^{5}$, confirmed that the presence of MWCNTs has a positive effect on MSCs cell proliferation and improve the biocompatibility behavior of the synthesized materials. 


\section{CONCLUSION}

The inorganic-polymeric composites designed in this work, appears to be good candidates as the material of choice for bone filler application, considering their characterization and biocompatibility properties. Further evaluations of the performance of MSCs on composites can be represented by in vitro cell culture under dynamic conditions and in vivo studies.

\section{ACKNOWLEDGEMENTS}

We thanks North Tehran branch, Islamic Azad University, Iran and Iranian Nanotechnology Initiative Council for providing the financial support of work.

\section{REFERENCES}

1. Rezaei, A.; Mohammadi, M.R. J. Materials Science and Engineering C. 2013, 33, 390396

2. Sadjadi, M. S.; Meskinfam, M.; Sadeghi, B.; Jazdarreh, H.; Zare, K. J. Biomedical Nanotechnology. 2011, 7, 450-454

3. Chen, Q. Z.; Harding, S. E.; Ali, N. N.; Lyon, A. R.; Boccaccini, A. R. J. Mater. Sci. Eng R-Rep. 2008, 59, 1-37

4. Nitesh R, P.; Piyush P, Gohil. J. Emerging Technology and Advanced Engineering. 2012, 2, 91-101.

5. Meskinfam, M.; Sadjadi, M. S.; Jazdarreh, H.; Zare, K. J. Biomedical Nanotechnology. 2011, 7, 455-459.

6. Kuttappan, S.; Mathew, D.; Nair, M. B. J. Biological macromolecules. 2016, 93(Pt B), 1390-1401.

7. Shi, S.; Chen, S.; Zhang, X.; Shen, W.; Li, X.; Hu, W.; Wang, H. J. Chem. Technol Biotechnol. 2009, 84, 285-290.

8. Zhang, X.; Bogdanowicz, D.; Erisken, C.; Lee, N. M.; Eng, M.; Lu, H. H. J. Shoulder Elbow Surg. 2012, 21, 266-277.

9. Sadjadi, M.S.; Meskinfam, M.; Sadeghi, B.; Jazdarreh, H.; Zare, K. J. Materials Chemistry and Physics. 2010, 124, 217-222.

10. Sinha, A.; Guha, A. J. Mater. Sci. Eng C. 2008, 29, 1330.

11. Meskinfam, M.; Sadjadi, M. S.; Jazdarreh, H. World Academy of Science, Engineering and Technology. 2011, 76, 394-398.

12. Rusa, V.; Ng, C.; Wilke, M.; Tiersch, B.; Fratzl, P.; Peter, M. J. Biomaterilas. 2005, 26, 54145426.

13. Chen, L.; Jingxiao, H.; Xinyu, S.; and Hua,
T. J. Mater Sci: Mater Med. 2013, 24, 18431851.

14. Peña, J.; Izquierdo-Barba, I.; Garcia, M. A.;Vallet-Regi, M. European Ceramic Society. 2006, 26, 3631-3638.

15. Mollazadeh, S.; Javadpour, J.; Khavandi, A. J. Ceramics Int. 2007, 33, 1579-83.

16. Mishra, R. K.; Majeed, A. B. A.; Banthia, A. K. Int J. Plas. Technol. 2011, 15, 82-95.

17. El, A.; Ali, H. J. Macromolecular science, part A: Pure and Applied chemistry. 2012, 49, 7-14.

18. Pulat, M.; Akalin, G. O.; Artificial Cells, Nanomedicine, and Biotechnolog. 2013, 41, 145-151.

19. Zhao, F.; Yin, Y.; Lu, W. W.; Chiyan Leong, J.; Zhang, W.; Zhang, J.; Zhang, M.; Yao, K. J. Biomaterials. 2002, 23, 3227-3234.

20. Shin, U. S.; Yoon, I. K.; Lee, G. S.; Jang, W. C.; Knowles, J. C.; Kim, H.W. J. Tissue Engineering. 2011, doi:10.4061/2011/674287.

21. Khare, R.; Bose, S. J. Minerals \& Materials Characterization \& Engineering. 2005, 4, 3146.

22. Salam, M. A.; Makki, M. S.; Abdelaal, M. Y. A. J. Alloys \& Compounds. 2011, 509, 25822587.

23. Vardharajula, S.; Ali, S. Z.; Tiwari, P. M.; Eroclu, E.; Vig, K.; Dennis, V. A.; Singh, S. R. J. Nanomedicine. 2012, 7, 5361-5374.

24. Yadav, S. K.; Bera, T.; Saxena, P. S.; Maurya, A. K.;Garbyal, R.S.;Vajtaei, R.; Ramachandrarao, P. Biomed Mater Res. A. 2010; 93, 886-896.

25. Zhao, B.; Hu, H.; Mandal, S. K.; Haddon, R. C. Chemistry of Materials. 2005, 17(12), 3235-3241. 